\title{
Some Common Fixed Point Theorems for Fuzzy Maps under Non-expansive Type Condition
}

\author{
R. D. Daheriya ${ }^{1}$, Umesh Dongre ${ }^{1 *}$, Manoj Ughade ${ }^{2}$ \\ ${ }^{1}$ Department of Mathematics, Government J.H. Post Graduate College, Betul, India \\ ${ }^{2}$ Department of Mathematics, Dr. B. R. A. Government College, Amla, India \\ ${ }^{3}$ Department of Mathematics, Sarvepalli Radhakrishnan University, Bhopal, India \\ *Correspondingauthor, e-mail: udhelpyou@gmail.com
}

\begin{abstract}
In this paper, we prove some common fixed point results for fuzzy mappings satisfying nonexpansive type condition.
\end{abstract}

Keywords: fuzzy mapping, common fixed point, linear metric space, non-expansive mapping.

\section{INTRODUCTION}

Let $(X, d)$ be a metric space and let $T$ be a self-mappings on $X$. If $T$ is such that for all $x, y$ in $X$

$$
d(T x, T y) \leq \lambda d(x, y)
$$

where $0<\lambda<1$, then $T$ is said to be a contraction mapping. If $T$ satisfies (1.1) with $\lambda=1$, then $T$ is called a non-expansive mapping. If $T$ satisfies any conditions of type

$$
d(T x, T y) \leq a_{1} d(x, y)+a_{2} d(x, T x)+a_{3} d(y, T y)+a_{4} d(x, T y)+a_{5} d(y, T x)
$$

where $a_{i}(i=1,2,3,4,5)$ are nonnegative real numbers such that $a_{1}+a_{2}+a_{3}+a_{4}+a_{5}<1$, then $\mathrm{T}$ is said to be a contractive type mapping. If $T$ satisfies (1.2) with $a_{1}+a_{2}+a_{3}+a_{4}+a_{5}=1$, then $\mathrm{T}$ is said to be a non-expansive type mapping. Similar terminology is used for multi-valued mappings.

Fixed point theorems for contractive, non-expansive, contractive type and non-expansive type mappings provide techniques for solving a variety of applied problems in mathematical and engineering sciences. It is one of the reason that many authors have studied various classes of contractive type or non-expansive type mappings. For Banach spaces the famous is Gregus's Fixed Point Theorem [10] for non-expansive type single-valued mappings, which satisfy (1.2) with $a_{4}=$ $a_{5}=0, a_{1}<1$. The class of mappings $T$ satisfying the following non-expansive type condition:

$$
\begin{aligned}
d(T x, T y) & \leq a(x, y) \max \left\{d(x, y), d(x, T x), d(y, T y), \frac{d(x, T x)+d(y, T y)}{2}\right\} \\
& +b(x, y) \max \{d(x, T x), d(y, T y)+c(x, y)[d(x, T y)+d(y, T x)]
\end{aligned}
$$

for all $x, y \in X$, where $a, b, c$ are nonnegative real numbers such that $b>0, c>0$ and $a+b+2 c=$ 1 , was introduced and investigated by Ciric [9]. Ciric proved that in a complete metric space such mappings have a unique fixed point. Chandra et al [7] consider the following generalization of (1.3), let $T, f: X \rightarrow X$ satisfying:

$$
\begin{aligned}
d(T x, T y) & \leq a(x, y) d(f x, f y)+b(x, y) \max \{d(f x, T x), d(f y, T y)\} \\
& +c(x, y)[d(f x, T y)+d(f y, T x)]
\end{aligned}
$$

where

$$
a(x, y) \geq 0, \quad \beta=\inf _{x, y \in X} b(x, y)>0, \gamma=\inf _{x, y \in X} c(x, y)>0
$$

with

$$
\sup _{x, y \in X}(a(x, y)+b(x, y)+2 c(x, y))=1 .
$$


Jhade et al [12] studied the following non-expansive type condition for two self-maps $T, f: X \rightarrow X$;

$$
\begin{aligned}
d(T x, T y) & \leq a(x, y) d(f x, f y)+b(x, y) \max \{d(f x, T x), d(f y, T y)\} \\
& +c(x, y) \max \{d(f x, f y), d(f x, T x), d(f y, T y)\} \\
& +e(x, y) \max \{d(f x, f y), d(f x, T x), d(f y, T y) d(f x, T y)\}
\end{aligned}
$$

where

$$
\begin{aligned}
& a(x, y), b(x, y), c(x, y), e(x, y) \geq 0, \\
& \beta=i n f_{x, y \in X} e(x, y)>0 \\
& \gamma=\inf _{x, y \in X}(1+b(x, y)+e(x, y))>0
\end{aligned}
$$

with

$$
\sup _{x, y \in X}(a(x, y)+b(x, y)+c(x, y)+2 e(x, y))=1 .
$$

In 1965, Zadeh [25] introduced the concept of a fuzzy set as a new way to represent vagueness in everyday life. The study of fixed point theorems in fuzzy mathematics was investigated by Weiss [24], Butnariu [5], Singh and Talwar [20], Mihet [14], Qiu et al. [16], and Beg and Abbas [2] and many others. Heilpern [11] first used the concept of fuzzy mappings to prove the Banach contraction principle for fuzzy (approximate quantity-valued) mappings on a complete metric linear spaces. The result obtained by Heilpern [11] is a fuzzy analogue of the fixed point theorem for multi-valued mappings of Nadler et al. [15]. Bose and Sahani [4], Vijayaraju and Marudai [21], improved the result of Heilpern. In some earlier work, Watson and Rhoades [22], [23] proved several fixed point theorems involving a very general contractive definition.

In this paper, we establish a common fixed point theorem for fuzzy maps satisfying non-expansive type condition on complete linear metric space. Also, a common fixed point theorem for sequence of fuzzy mappings satisfying non-expansive type condition.

\section{Preliminaries}

In this paper, we shall generally follow the notations of Heilpern [11].

Definition 2.1 Let $(X, d)$ be a complete linear metric space and $\mathcal{F}(X)$, the collection of all fuzzy sets in $X$. A fuzzy set in $X$ is a function with domain $X$ and values in [0,1]. If $A$ is a fuzzy set and $x \in X$, then the function value $A(x)$ is called the grade of membership of $x$ in $A$. The $\alpha$-level set of $A$ is denoted by

$$
\begin{aligned}
& A_{\alpha}=\{x: A(x) \geq \alpha\} \text { if } \alpha \in(0,1] \\
& A_{0}=\overline{\{x: A(x)>0\},}
\end{aligned}
$$

where $\bar{B}$ stands for the (non-fuzzy) closure of a set $B$.

Definition 2.2 A fuzzy set $A$ is said to be an approximate quantity if and only if $A_{\alpha}$ is compact and convex for each $\alpha \in(0,1]$ and $\sup _{x \in X} A(x)=1$, when $A$ is an approximate quantity and $A\left(x_{0}\right)=$ 1 for some $x_{0} \in X, A$ is identified with an approximation of $x_{0}$. From the collection $\mathcal{F}(X)$, a subcollection of all appropriate quantities is denoted as $\mathcal{W}(X)$.

Definition 2.3 The distance between two appropriate quantities is defined by the following scheme. Let $A, B \in \mathcal{W}(X)$ and $\alpha \in[0,1]$,

$$
\begin{aligned}
& D_{\alpha}(A, B)=\inf _{x \in A_{\alpha}, y \in B_{\alpha}} d(x, y) ; \\
& H_{\alpha}(A, B)=\operatorname{dist} d\left(A_{\alpha}, B_{\alpha}\right) ; \\
& H(A, B)=\sup _{\alpha} D_{\alpha}(A, B) ;
\end{aligned}
$$

wherein the dist is in the sense of Hausdorff distance .The function $D_{\alpha}$ is called an $\alpha$-distance (induced by $d$ ), $H_{\alpha}$ a $\alpha$-distance (induced by dist) and $H$ a distance between $A$ and $B$. Note that $D_{\alpha}$ is a non-decreasing function of $\alpha$. 
Definition 2.4 Let $A, B \in \mathcal{W}(X)$. Then $A$ is said to be more accurate than $B$, denoted by $A \subset B$, iff $A(x) \leq B(x)$ for each $x \in X$. The relation $\subset$ induces a partial ordering on the family $\mathcal{W}(X)$.

Definition 2.5 Let $Y$ be an arbitrary set and $X$ be any metric space. $F$ is called a fuzzy mapping if and only if $F$ is a mapping from the set $Y$ into $\mathcal{W}(X)$. A fuzzy mapping $F$ is a fuzzy subset of $Y \times X$ with membership function $F(y, x)$. The function value $F(y, x)$ is the grade of membership of $x$ in $F(y)$. Note that each fuzzy mapping is a set valued mapping. Let $A \in F(X), B \in F(Y)$. Then he fuzzy set $F(A)$ in $F(X)$ is defined by

$$
F(A)(x)=\sup _{y \in X}(F(y, x) \wedge A(y)), \mathrm{x} \in \mathrm{X}
$$

and the fuzzy set $F^{-1}(B)$ in $F(Y)$ is defined by

$$
F^{-1}(B)(y)=\sup _{x \in X} F(y, x) \wedge B(x), \mathrm{y} \in \mathrm{Y}
$$

Lee [13] proved the following.

Lemma 2.6 Let $(X, d)$ be a complete linear metric space, $F$ is a fuzzy mapping from $X$ into $\mathcal{W}(X)$ and $x_{0} \in X$, then there exists an $x_{1} \in X$ such that $\left\{x_{1}\right\} \subset F\left(x_{0}\right)$.

The following two lemmas are due to Heilpern [11].

Lemma 2.7 Let $x \in X, A \in \mathcal{W}(X)$ and $\{x\}$ a fuzzy set with membership function equal to a characteristic function of $\{x\}$. If $\{x\} \subset A$, then $D_{\alpha}(x, A)=0$ for each $\alpha \in[0,1]$.

Lemma 2.8 Let $A, B \in \mathcal{W}(X), \alpha \in[0,1]$ and $D_{\alpha}(A, B)=\inf _{x \in A_{\alpha}, y \in B_{\alpha}} d(x, y)$, where $A_{\alpha}=$ $\{x: A(x) \geq \alpha\}$, then

$$
D_{\alpha}(x, A) \leq d(x, y)+D_{\alpha}(y, A)
$$

for each $x, y \in X$.

Lemma 2.9 Let $H_{\alpha}(A, B)=\operatorname{dist} d\left(A_{\alpha}, B_{\alpha}\right)$, where 'dist' is the Hausdorff distance. If $\left\{x_{0} \subset A\right\}$, then $D_{\alpha}\left(x_{0}, B\right) \leq H_{\alpha}(A, B)$ for each $B \in \mathcal{W}(X)$.

Rhoades [18] proved the following common fixed point theorem involving a very general contractive condition, for fuzzy mappings on complete linear metric space. He proved the following theorem.

Theorem 2.10 Let $(X, d)$ be a complete linear metric space and let $F, G$ be fuzzy mappings from $X$ into $\mathcal{W}(X)$ satisfying

$$
H(F x, G y) \leq Q(m(x, y)), \text { for all } x, y \in X,
$$

where

$$
m(x, y)=\max \left\{d(x, y), D_{\alpha}(x, F x), D_{\alpha}(y, G y), \frac{D_{\alpha}(x, G y)+D_{\alpha}(y, F x)}{2}\right\}
$$

and $Q$ is a real-valued function defined on $D$, the closure of the range of $d$, satisfying the following three conditions:

a) $0<Q(s)<s$ for each $s \in D \backslash\{0\}$ and $Q(0)=0$,

b) $Q$ is non-decreasing on $D$, and

c) $g(s)=s / s-Q(s)$ is non-increasing on $D \backslash\{0\}$.

Then there exists a point $z$ in $X$ such that $\{z\} \subset F z \cap G z$.

In [17] Rhoades, generalized the result of Theorem 2.10 for sequence of fuzzy mappings on complete linear metric space. He proved the following theorem.

Theorem 2.11 Let $g$ be a non-expansive self-mapping of a complete linear metric space $(X, d)$ and $\left\{F_{i}\right\}$ be a sequence of fuzzy mappings from $X$ into $W(X)$. For each pair of fuzzy mappings $F_{i}, F_{j}$ and for any $x \in X,\left\{u_{x}\right\} \subset F_{i}(x)$, there exists a $\left\{v_{y}\right\} \subset F_{j}(y)$ for all $y \in X$ such that

$$
D\left(\left\{u_{x}\right\},\left\{v_{y}\right\}\right) \leq Q(m(x, y)) \text {, for all } x, y \in X,
$$

where 


$$
m(x, y)=\max \left\{(g(x), g(y)), d\left(g(x), g\left(u_{x}\right)\right), d\left(g(y), g\left(v_{y}\right)\right), \frac{d\left(g(x), g\left(v_{y}\right)\right)+d\left(g(y), g\left(u_{x}\right)\right)}{2}\right\}
$$

and $Q$ satisfying the conditions (a)-(c) of Theorem 2.10. Then there exists $\{z\} \subset \bigcap_{i=1}^{\infty} F_{i}(z)$

\section{Main Results}

Now, we give our first main result.

Theorem 3.1 Let $(X, d)$ be a complete linear metric space. $F$ and $G$ are two fuzzy mappings from $X$ into $\mathcal{W}(X)$ satisfying:

$$
\begin{aligned}
H(F x, G y) & \leq a(x, y) d(x, y)+b(x, y) \max \left\{D_{\alpha}(x, F x), D_{\alpha}(y, G y)\right\} \\
& +c(x, y) \max \left\{d(x, y), D_{\alpha}(x, F x), D_{\alpha}(y, G y)\right\} \\
& +e(x, y) \max \left\{d(x, y), D_{\alpha}(x, F x), D_{\alpha}(y, G y), D_{\alpha}(x, G y)\right\} \\
& +h(x, y) \max \left\{d(x, y), D_{\alpha}(x, F x), D_{\alpha}(y, G y), D_{\alpha}(x, G y), D_{\alpha}(y, F x)\right\}
\end{aligned}
$$

where $a(x, y), b(x, y), c(x, y), e(x, y), h(x, y)$ are non-negative real functions from $X \times X$ into $[0,+\infty)$ such that

$$
\begin{gathered}
\beta=i n f_{x, y \in X}(e(x, y)+h(x, y))>0 \\
\gamma=\inf _{x, y \in X}(b(x, y)+e(x, y)+h(x, y))>0
\end{gathered}
$$

with

$$
\sup _{x, y \in X}(a(x, y)+b(x, y)+c(x, y)+2 e(x, y)+2 h(x, y))=1 .
$$

Then there exists a point $z$ in $X$, which is a common fixed point of $F$ and $G$, i.e. $\{z\} \subset F z \cap G z$.

Proof. Pick $x_{0}$ in $X$, then by Lemma 2.6, we can choose $x_{1} \in X$ such that $\left\{x_{1}\right\} \subset F x_{0}$. Choose $x_{2} \in X$ such that $\left\{x_{2}\right\} \subset G x_{1}$ and $d\left(x_{1}, x_{2}\right) \leq H\left(F x_{0}, G x_{1}\right)$. Continuing the process, we obtain a sequence $\left\{x_{n}\right\}$ such that $\left\{x_{2 n+1}\right\} \subset F x_{2 n},\left\{x_{2 n+2}\right\} \subset G x_{2 n+1}$ such that $d\left(x_{2 n+1}, x_{2 n+2}\right) \leq H\left(F x_{2 n}, G x_{2 n+1}\right)$, where $n=0,1,2, \ldots$ Applying (3.1) and using triangle inequality, we have

$$
\begin{aligned}
d\left(x_{2 n+1}, x_{2 n+2}\right) & \leq H\left(F x_{2 n}, G x_{2 n+1}\right) \\
\leq & \operatorname{ad}\left(x_{2 n}, x_{2 n+1}\right)+b \max \left\{D_{\alpha}\left(x_{2 n}, F x_{2 n}\right), D_{\alpha}\left(x_{2 n+1}, G x_{2 n+1}\right)\right\} \\
+ & c \max \left\{d\left(x_{2 n}, x_{2 n+1}\right), D_{\alpha}\left(x_{2 n}, F x_{2 n}\right), D_{\alpha}\left(x_{2 n+1}, G x_{2 n+1}\right)\right\} \\
+ & e \max \left\{d\left(x_{2 n}, x_{2 n+1}\right), D_{\alpha}\left(x_{2 n}, F x_{2 n}\right), D_{\alpha}\left(x_{2 n+1}, G x_{2 n+1}\right)\right. \\
& \left., D_{\alpha}\left(x_{2 n}, G x_{2 n+1}\right)\right\} \\
& +h \max \left\{d\left(x_{2 n}, x_{2 n+1}\right), D_{\alpha}\left(x_{2 n}, F x_{2 n}\right), D_{\alpha}\left(x_{2 n+1}, G x_{2 n+1}\right)\right. \\
& \left., D_{\alpha}\left(x_{2 n}, G x_{2 n+1}\right), D_{\alpha}\left(x_{2 n+1}, F x_{2 n}\right)\right\} \\
\leq & \operatorname{ad}\left(x_{2 n}, x_{2 n+1}\right)+b \max \left\{d\left(x_{2 n}, x_{2 n+1}\right), d\left(x_{2 n+1}, x_{2 n+2}\right)\right\} \\
+ & c \max \left\{d\left(x_{2 n}, x_{2 n+1}\right), d\left(x_{2 n}, x_{2 n+1}\right), d\left(x_{2 n+1}, x_{2 n+2}\right)\right\} \\
+ & e \max \left\{d\left(x_{2 n}, x_{2 n+1}\right), d\left(x_{2 n}, x_{2 n+1}\right), d\left(x_{2 n+1}, x_{2 n+2}\right), d\left(x_{2 n}, x_{2 n+2}\right)\right\} \\
+ & h \max \left\{d\left(x_{2 n}, x_{2 n+1}\right), d\left(x_{2 n}, x_{2 n+1}\right), d\left(x_{2 n+1}, x_{2 n+2}\right), d\left(x_{2 n}, x_{2 n+2}\right)\right. \\
, & \left.d\left(x_{2 n+1}, x_{2 n+1}\right)\right\} \\
& \leq a d\left(x_{2 n}, x_{2 n+1}\right)+(b+c) \max \left\{d\left(x_{2 n}, x_{2 n+1}\right), d\left(x_{2 n+1}, x_{2 n+2}\right)\right\} \\
+ & (e+h) \max \left\{d\left(x_{2 n}, x_{2 n+1}\right), d\left(x_{2 n+1}, x_{2 n+2}\right)\right. \\
, & \left.d\left(x_{2 n}, x_{2 n+1}\right)+d\left(x_{2 n+1}, x_{2 n+2}\right)\right\}
\end{aligned}
$$

where $a, b, c, e$ and $h$ are evaluated at $\left(x_{2 n}, x_{2 n+1}\right)$. 
If for some $n, d\left(x_{2 n+1}, x_{2 n+2}\right)>d\left(x_{2 n}, x_{2 n+1}\right)$. The last inequality gives

$$
d\left(x_{2 n+1}, x_{2 n+2}\right)<(a+b+c+2 e+2 h) d\left(x_{2 n}, x_{2 n+1}\right)
$$

a contradiction. Therefore, for all $n$, we have

$$
d\left(x_{2 n+1}, x_{2 n+2}\right) \leq d\left(x_{2 n}, x_{2 n+1}\right)
$$

Hence, for all positive integers $n$,

$$
d\left(x_{2 n+1}, x_{2 n+2}\right) \leq d\left(x_{0}, x_{1}\right)
$$

Again applying (3.1) and triangle inequality, we have

$$
\begin{aligned}
d\left(x_{2}, x_{3}\right) & \leq H\left(F x_{1}, G x_{2}\right) \\
& \leq \operatorname{ad}\left(x_{1}, x_{2}\right)+b \max \left\{D_{\alpha}\left(x_{1}, F x_{1}\right), D_{\alpha}\left(x_{2}, G x_{2}\right)\right\} \\
& +c \max \left\{d\left(x_{1}, x_{2}\right), D_{\alpha}\left(x_{1}, F x_{1}\right), D_{\alpha}\left(x_{2}, G x_{2}\right)\right\} \\
& +e \max \left\{d\left(x_{1}, x_{2}\right), D_{\alpha}\left(x_{1}, F x_{1}\right), D_{\alpha}\left(x_{2}, G x_{2}\right), D_{\alpha}\left(x_{1}, G x_{2}\right)\right\} \\
& +h \max \left\{d\left(x_{1}, x_{2}\right), D_{\alpha}\left(x_{1}, F x_{1}\right), D_{\alpha}\left(x_{2}, G x_{2}\right), D_{\alpha}\left(x_{1}, G x_{2}\right)\right. \\
& \left., D_{\alpha}\left(x_{2}, F x_{1}\right)\right\} \\
& \leq \operatorname{ad}\left(x_{1}, x_{2}\right)+b \max \left\{d\left(x_{1}, x_{2}\right), d\left(x_{2}, x_{3}\right)\right\} \\
& +c \max \left\{d\left(x_{1}, x_{2}\right), d\left(x_{1}, x_{2}\right), d\left(x_{2}, x_{3}\right)\right\} \\
& +e \max \left\{d\left(x_{1}, x_{2}\right), d\left(x_{1}, x_{2}\right), d\left(x_{2}, x_{3}\right), d\left(x_{1}, x_{3}\right)\right\} \\
& +h \max \left\{d\left(x_{1}, x_{2}\right), d\left(x_{1}, x_{2}\right), d\left(x_{2}, x_{3}\right), d\left(x_{1}, x_{3}\right), d\left(x_{2}, x_{2}\right)\right\}
\end{aligned}
$$

where $a, b, c, e$ and $h$ are evaluated at $\left(x_{1}, x_{2}\right)$. Using (3.5), we have

$$
\begin{aligned}
d\left(x_{2}, x_{3}\right) & \leq \operatorname{ad}\left(x_{0}, x_{1}\right)+b \max \left\{d\left(x_{0}, x_{1}\right), d\left(x_{0}, x_{1}\right)\right\} \\
& +c \max \left\{d\left(x_{0}, x_{1}\right), d\left(x_{0}, x_{1}\right), d\left(x_{0}, x_{1}\right)\right\} \\
& +e \max \left\{d\left(x_{0}, x_{1}\right), d\left(x_{0}, x_{1}\right), d\left(x_{0}, x_{1}\right), d\left(x_{1}, x_{3}\right)\right\} \\
& +h \max \left\{d\left(x_{0}, x_{1}\right), d\left(x_{0}, x_{1}\right), d\left(x_{0}, x_{1}\right), d\left(x_{1}, x_{3}\right)\right\} \\
& =(a+b+c) d\left(x_{0}, x_{1}\right) \\
& +(e+h) \max \left\{d\left(x_{0}, x_{1}\right), d\left(x_{1}, x_{3}\right)\right\}
\end{aligned}
$$

Applying (3.1) again, we have

$$
\begin{aligned}
d\left(x_{1}, x_{3}\right) & \leq H\left(F x_{0}, G x_{2}\right) \\
& \leq \operatorname{ad}\left(x_{0}, x_{2}\right)+b \max \left\{D_{\alpha}\left(x_{0}, F x_{0}\right), D_{\alpha}\left(x_{2}, G x_{2}\right)\right\} \\
& +c \max \left\{d\left(x_{0}, x_{2}\right), D_{\alpha}\left(x_{0}, F x_{0}\right), D_{\alpha}\left(x_{2}, G x_{2}\right)\right\} \\
& +e \max \left\{d\left(x_{0}, x_{2}\right), D_{\alpha}\left(x_{0}, F x_{0}\right), D_{\alpha}\left(x_{2}, G x_{2}\right), D_{\alpha}\left(x_{0}, G x_{2}\right)\right\} \\
& +h \max \left\{d\left(x_{0}, x_{2}\right), D_{\alpha}\left(x_{0}, F x_{0}\right), D_{\alpha}\left(x_{2}, G x_{2}\right), D_{\alpha}\left(x_{0}, G x_{2}\right), D_{\alpha}\left(x_{2}, F x_{0}\right)\right\} \\
& \leq \operatorname{ad}\left(x_{0}, x_{2}\right)+b \max \left\{d\left(x_{0}, x_{1}\right), d\left(x_{2}, x_{3}\right)\right\} \\
& +c \max \left\{d\left(x_{0}, x_{2}\right), d\left(x_{0}, x_{1}\right), d\left(x_{2}, x_{3}\right)\right\} \\
& +e \max \left\{d\left(x_{0}, x_{2}\right), d\left(x_{0}, x_{1}\right), d\left(x_{2}, x_{3}\right), d\left(x_{0}, x_{3}\right)\right\} \\
& +h \max \left\{d\left(x_{0}, x_{2}\right), d\left(x_{0}, x_{1}\right), d\left(x_{2}, x_{3}\right), d\left(x_{0}, x_{3}\right), d\left(x_{2}, x_{1}\right)\right\}
\end{aligned}
$$

where $a, b, c, e$ and $h$ are evaluated at $\left(x_{0}, x_{2}\right)$. Since

$$
\begin{aligned}
d\left(x_{0}, x_{2}\right) & \leq d\left(x_{0}, x_{1}\right)+d\left(x_{1}, x_{2}\right) \leq 2 d\left(x_{0}, x_{1}\right) \\
d\left(x_{0}, x_{3}\right) & \leq d\left(x_{0}, x_{1}\right)+d\left(x_{1}, x_{3}\right) \\
& \leq d\left(x_{0}, x_{1}\right)+d\left(x_{1}, x_{2}\right)+d\left(x_{2}, x_{3}\right)
\end{aligned}
$$




$$
\leq 3 d\left(x_{0}, x_{1}\right)
$$

Using (3.5) and (3.7), we have

$$
d\left(x_{1}, x_{3}\right) \leq(2 a+b+2 c+3 e+3 h) d\left(x_{0}, x_{1}\right)
$$

Implies that

$$
d\left(x_{1}, x_{3}\right) \leq(2-b-e-h) d\left(x_{0}, x_{1}\right)
$$

Hence, from (3.7)

$$
\begin{aligned}
d\left(x_{2}, x_{3}\right) & \leq a d\left(x_{0}, x_{1}\right)+b d\left(x_{0}, x_{1}\right)+c d\left(x_{0}, x_{1}\right) \\
& +(e+h)(2-b-e-h) d\left(x_{0}, x_{1}\right) \\
& =(a+b+c+(e+h)(2-b-e-h)) d\left(x_{0}, x_{1}\right) \\
& =(1-(e+h)(b+e+h)) d\left(x_{0}, x_{1}\right) \\
& \leq(1-\beta \gamma) d\left(x_{0}, x_{1}\right)
\end{aligned}
$$

It is easy to show that

$$
d\left(x_{n}, x_{n+1}\right) \leq(1-\beta \gamma)^{\left[\frac{n}{2}\right]} d\left(x_{0}, x_{1}\right)
$$

where $\left[\frac{n}{2}\right]$ stands for the greatest integer not exceeding $\frac{n}{2}$. Also, since $\beta \gamma>0$, from (3.8), we have $\left\{x_{n}\right\}_{n \in \mathbb{N}}$ is a Cauchy sequence in $X$. Since $X$ is complete, there is a point $z \in X$ such that

$$
\lim _{n \rightarrow+\infty} x_{n}=z \text {. }
$$

Since $\alpha \in[0,1]$, then using Lemma 2.8 and Lemma 2.9, we have

$$
\begin{aligned}
D_{\alpha}(z, F z) & \leq d\left(z, G x_{n}\right)+D_{\alpha}\left(G x_{n}, F z\right) \\
& \leq d\left(z, G x_{n}\right)+H_{\alpha}\left(F z, G x_{n}\right) \\
& \leq d\left(z, G x_{n}\right)+H\left(F z, G x_{n}\right)
\end{aligned}
$$

Taking limit as $n \rightarrow+\infty$, we get

$$
D_{\alpha}(z, F z) \leq \lim _{n \rightarrow+\infty} D_{\alpha}\left(F z, G x_{n}\right) \leq \lim _{n \rightarrow+\infty} H\left(F z, G x_{n}\right)
$$

Again from (3.1), we have

$$
\begin{aligned}
H\left(F z, G x_{n}\right) & \leq \operatorname{ad}\left(z, x_{n}\right)+b \max \left\{D_{\alpha}(z, F z), D_{\alpha}\left(x_{n}, G x_{n}\right)\right\} \\
& +c \max \left\{d\left(z, x_{n}\right), D_{\alpha}(z, F z), D_{\alpha}\left(x_{n}, G x_{n}\right)\right\} \\
& +e \max \left\{d\left(z, x_{n}\right), D_{\alpha}(z, F z), D_{\alpha}\left(x_{n}, G x_{n}\right), D_{\alpha}\left(z, G x_{n}\right)\right\} \\
& +h \max \left\{d\left(z, x_{n}\right), D_{\alpha}(z, F z), D_{\alpha}\left(x_{n}, G x_{n}\right), D_{\alpha}\left(z, G x_{n}\right), D_{\alpha}\left(x_{n}, F z\right)\right\} \\
& \leq \sup _{x, y \in X}(a+b+c+e+h) \max \left\{d\left(z, x_{n}\right), \max \left\{D_{\alpha}(z, F z), D_{\alpha}\left(x_{n}, G x_{n}\right)\right\}\right. \\
& , \max \left\{d\left(z, x_{n}\right), D_{\alpha}(z, F z), D_{\alpha}\left(x_{n}, G x_{n}\right)\right\} \\
& , \max \left\{d\left(z, x_{n}\right), D_{\alpha}(z, F z), D_{\alpha}\left(x_{n}, G x_{n}\right), D_{\alpha}\left(z, G x_{n}\right)\right\} \\
& , \max \left\{d\left(z, x_{n}\right), D_{\alpha}(z, F z), D_{\alpha}\left(x_{n}, G x_{n}\right), D_{\alpha}\left(z, G x_{n}\right), D_{\alpha}\left(x_{n}, F z\right)\right\}
\end{aligned}
$$

Letting limit as $n \rightarrow+\infty$, we get

$$
\lim _{n \rightarrow+\infty} H\left(F z, G x_{n}\right) \leq \sup _{x, y \in X}(a+b+c+e+h) D_{\alpha}(z, F z)=D_{\alpha}(z, F z)
$$

Using (3.10) and (3.11), we have

$$
D_{\alpha}(z, F z) \leq D_{\alpha}(z, F z)
$$

a contradiction. Hence we must have $D_{\alpha}(z, F z)=0$. Since $\alpha$ is arbitrary number in [0,1]. It follows that $D(z, F z)=0$, which implies that $\{z\} \subset F z$. Similarly it can be shown that $\{z\} \subset G z$. Hence $\{z\} \subset F z \cap G z$. 
Now, we prove a common fixed point theorem for sequence of fuzzy mappings of non-expansive condition.

Theorem 3.2 Let $g$ be a non-expansive self-mapping of a complete linear metric space $(X, d)$ and $\left\{F_{i}\right\}$ be a sequence of fuzzy mappings from $X$ into $\mathcal{W}(X)$. For each pair of fuzzy mappings $F_{i}, F_{j}$ and for any $x \in X,\left\{u_{x}\right\} \subset F_{i}(x)$, there exists a $\left\{v_{y}\right\} \subset F_{j}(y)$ for all $y \in X$ such that

$$
\begin{aligned}
D\left(\left\{u_{x}\right\},\left\{v_{y}\right\}\right) & \leq \operatorname{ad}(g(x), g(y))+b \max \left\{d\left(g(x), g\left(u_{x}\right)\right), d\left(g(y), g\left(v_{y}\right)\right)\right\} \\
& +c \max \left\{(g(x), g(y)), d\left(g(x), g\left(u_{x}\right)\right), d\left(g(y), g\left(v_{y}\right)\right)\right\} \\
& +e \max \left\{(g(x), g(y)), d\left(g(x), g\left(u_{x}\right)\right), d\left(g(y), g\left(v_{y}\right)\right), d\left(g(x), g\left(v_{y}\right)\right)\right\} \\
& +h \max \left\{(g(x), g(y)), d\left(g(x), g\left(u_{x}\right)\right), d\left(g(y), g\left(v_{y}\right)\right), d\left(g(x), g\left(v_{y}\right)\right)\right. \\
& \left., d\left(g(y), g\left(u_{x}\right)\right)\right\}
\end{aligned}
$$

where $a, b, c, d, e$ are non-negative real number such that $\beta=e+h>0$ and $\gamma=b+e+h>0$ with $a+b+c+2 e+2 h=1$. Then there exists a point $z$ in $X$, which is a common fixed point of sequence of fuzzy mappings, i.e. $\{z\} \subset \bigcap_{i=1}^{\infty} F_{i}(z)$.

Proof. Choose $x_{0} \in X$, then by Lemma 2.6, we can choose $x_{1} \in X$ such that $\left\{x_{1}\right\} \subset F\left(x_{0}\right)$. From the hypothesis, there exists an $x_{2} \in X$ such that $\left\{x_{2}\right\} \subset F\left(x_{1}\right)$. In general, choose $x_{n+1} \in X$ such that $\left\{x_{n+1}\right\} \subset F_{n+1}\left(x_{n}\right)$.

Applying (3.12), we have

$$
\begin{aligned}
D\left(\left\{x_{n}\right\},\left\{x_{n+1}\right\}\right) & \leq \operatorname{ad}\left(g\left(x_{n-1}\right), g\left(x_{n}\right)\right) \\
& +b \max \left\{d\left(g\left(x_{n-1}\right), g\left(x_{n}\right)\right), d\left(g\left(x_{n}\right), g\left(x_{n+1}\right)\right)\right\} \\
& +c \max \left\{d\left(g\left(x_{n-1}\right), g\left(x_{n}\right)\right), d\left(g\left(x_{n-1}\right), g\left(x_{n}\right)\right), d\left(g\left(x_{n}\right), g\left(x_{n+1}\right)\right)\right\} \\
& +e \max \left\{d\left(g\left(x_{n-1}\right), g\left(x_{n}\right)\right), d\left(g\left(x_{n-1}\right), g\left(x_{n}\right)\right), d\left(g\left(x_{n}\right), g\left(x_{n+1}\right)\right)\right. \\
& \left., d\left(g\left(x_{n-1}\right), g\left(x_{n+1}\right)\right)\right\} \\
& +h \max \left\{d\left(g\left(x_{n-1}\right), g\left(x_{n}\right)\right), d\left(g\left(x_{n-1}\right), g\left(x_{n}\right)\right), d\left(g\left(x_{n}\right), g\left(x_{n+1}\right)\right)\right. \\
& \left., d\left(g\left(x_{n-1}\right), g\left(x_{n+1}\right)\right), d\left(g\left(x_{n}\right), g\left(x_{n}\right)\right)\right\}
\end{aligned}
$$

Since $g$ is a non-expansive self-mapping and $D\left(\left\{x_{n}\right\},\left\{x_{n+1}\right\}\right)=d\left(x_{n}, x_{n+1}\right)$, we get

$$
\begin{aligned}
& d\left(x_{n}, x_{n+1}\right)=D\left(\left\{x_{n}\right\},\left\{x_{n+1}\right\}\right) \\
& \quad \leq \operatorname{ad}\left(x_{n-1}, x_{n}\right)+b \max \left\{d\left(x_{n-1}, x_{n}\right), d\left(x_{n}, x_{n+1}\right)\right\} \\
& \quad+c \max \left\{d\left(x_{n-1}, x_{n}\right), d\left(x_{n-1}, x_{n}\right), d\left(x_{n}, x_{n+1}\right)\right\} \\
& \quad+e \max \left\{d\left(x_{n-1}, x_{n}\right), d\left(x_{n-1}, x_{n}\right), d\left(x_{n}, x_{n+1}\right), d\left(x_{n-1}, x_{n+1}\right)\right\} \\
& \quad+h \max \left\{d\left(x_{n-1}, x_{n}\right), d\left(x_{n-1}, x_{n}\right), d\left(x_{n}, x_{n+1}\right), d\left(x_{n-1}, x_{n+1}\right), d\left(x_{n}, x_{n}\right)\right\}
\end{aligned}
$$

If $d\left(x_{n-1}, x_{n}\right)<d\left(x_{n}, x_{n+1}\right)$ for some $n$, then by using triangle inequality, the last inequality gives

$$
d\left(x_{n}, x_{n+1}\right) \leq(a+b+c+2 e+2 h) d\left(x_{n}, x_{n+1}\right)
$$

a contradiction. Thus $d\left(x_{n}, x_{n+1}\right) \leq d\left(x_{n-1}, x_{n}\right)$. Hence, for all positive integers $n$,

$$
d\left(x_{n}, x_{n+1}\right) \leq d\left(x_{0}, x_{1}\right)
$$

Again applying (3.12) and using (3.13), we have

$$
\begin{aligned}
D\left(\left\{x_{2}\right\},\left\{x_{3}\right\}\right) & \leq \operatorname{ad}\left(g\left(x_{1}\right), g\left(x_{2}\right)\right)+b \max \left\{d\left(g\left(x_{1}\right), g\left(x_{2}\right)\right), d\left(g\left(x_{2}\right), g\left(x_{3}\right)\right)\right\} \\
& +c \max \left\{d\left(g\left(x_{1}\right), g\left(x_{2}\right)\right), d\left(g\left(x_{1}\right), g\left(x_{2}\right)\right), d\left(g\left(x_{2}\right), g\left(x_{3}\right)\right)\right\} \\
& +e \max \left\{d\left(g\left(x_{1}\right), g\left(x_{2}\right)\right), d\left(g\left(x_{1}\right), g\left(x_{2}\right)\right), d\left(g\left(x_{2}\right), g\left(x_{3}\right)\right)\right.
\end{aligned}
$$




$$
\begin{aligned}
& \left., d\left(g\left(x_{1}\right), g\left(x_{3}\right)\right)\right\} \\
& +h \max \left\{d\left(g\left(x_{1}\right), g\left(x_{2}\right)\right), d\left(g\left(x_{1}\right), g\left(x_{2}\right)\right), d\left(g\left(x_{2}\right), g\left(x_{3}\right)\right)\right. \\
& \left., d\left(g\left(x_{1}\right), g\left(x_{3}\right)\right), d\left(g\left(x_{2}\right), g\left(x_{2}\right)\right)\right\}
\end{aligned}
$$

Since $g$ is a non-expansive self-mapping and $D\left(\left\{x_{2}\right\},\left\{x_{3}\right\}\right)=d\left(x_{2}, x_{3}\right)$, we get

$$
\begin{aligned}
d\left(x_{2}, x_{3}\right) & =D\left(\left\{x_{2}\right\},\left\{x_{3}\right\}\right) \\
& \leq \operatorname{ad}\left(x_{1}, x_{2}\right)+(b+c) \max \left\{d\left(x_{1}, x_{2}\right), d\left(x_{2}, x_{3}\right)\right\} \\
& +(e+h) \max \left\{d\left(x_{1}, x_{2}\right), d\left(x_{2}, x_{3}\right), d\left(x_{1}, x_{3}\right)\right\}
\end{aligned}
$$

Again applying (3.12), we have

$$
\begin{aligned}
D\left(\left\{x_{1}\right\},\left\{x_{3}\right\}\right) & \leq \operatorname{ad}\left(g\left(x_{0}\right), g\left(x_{2}\right)\right)+b \max \left\{d\left(g\left(x_{0}\right), g\left(x_{2}\right)\right), d\left(g\left(x_{2}\right), g\left(x_{3}\right)\right)\right\} \\
& +c \max \left\{d\left(g\left(x_{0}\right), g\left(x_{2}\right)\right), d\left(g\left(x_{0}\right), g\left(x_{2}\right)\right), d\left(g\left(x_{2}\right), g\left(x_{3}\right)\right)\right\} \\
& +e \max \left\{d\left(g\left(x_{0}\right), g\left(x_{2}\right)\right), d\left(g\left(x_{0}\right), g\left(x_{2}\right)\right), d\left(g\left(x_{2}\right), g\left(x_{3}\right)\right)\right. \\
& \left., d\left(g\left(x_{0}\right), g\left(x_{3}\right)\right)\right\} \\
& +h \max \left\{d\left(g\left(x_{0}\right), g\left(x_{2}\right)\right), d\left(g\left(x_{0}\right), g\left(x_{2}\right)\right), d\left(g\left(x_{2}\right), g\left(x_{3}\right)\right)\right. \\
& \left., d\left(g\left(x_{0}\right), g\left(x_{3}\right)\right), d\left(g\left(x_{2}\right), g\left(x_{2}\right)\right)\right\}
\end{aligned}
$$

Since $g$ is a non-expansive self-mapping and $D\left(\left\{x_{1}\right\},\left\{x_{3}\right\}\right)=d\left(x_{1}, x_{3}\right)$. By using (3.13) and triangle inequality, we get

$$
\begin{aligned}
d\left(x_{2}, x_{3}\right) & =D\left(\left\{x_{2}\right\},\left\{x_{3}\right\}\right) \\
\leq & \operatorname{ad}\left(x_{0}, x_{2}\right)+(b+c) \max \left\{d\left(x_{0}, x_{2}\right), d\left(x_{2}, x_{3}\right)\right\} \\
+ & (e+h) \max \left\{d\left(x_{0}, x_{2}\right), d\left(x_{2}, x_{3}\right), d\left(x_{0}, x_{3}\right)\right\} \\
\leq & \operatorname{ad}\left(x_{0}, x_{2}\right)+(b+c) \max \left\{d\left(x_{0}, x_{2}\right), d\left(x_{2}, x_{3}\right)\right\} \\
+ & (e+h) \max \left\{d\left(x_{0}, x_{2}\right), d\left(x_{2}, x_{3}\right), d\left(x_{0}, x_{3}\right)\right\} \\
\leq & (2 a+b+2 c+3 e+3 h) d\left(x_{0}, x_{1}\right) \\
& =(2-b-e-h) d\left(x_{0}, x_{1}\right)
\end{aligned}
$$

Hence, from (3.14) and (3.15), we have

$$
\begin{aligned}
d\left(x_{2}, x_{3}\right) & \leq a d\left(x_{0}, x_{1}\right)+b d\left(x_{0}, x_{1}\right)+c d\left(x_{0}, x_{1}\right) \\
& +(e+h)(2-b-e-h) d\left(x_{0}, x_{1}\right) \\
& =(a+b+c+(e+h)(2-b-e-h)) d\left(x_{0}, x_{1}\right) \\
& =(1-(e+h)(b+e+h)) d\left(x_{0}, x_{1}\right) \\
& \leq(1-\beta \gamma) d\left(x_{0}, x_{1}\right)
\end{aligned}
$$

It is easy to show that

$$
d\left(x_{n}, x_{n+1}\right) \leq(1-\beta \gamma)^{\left[\frac{n}{2}\right]} d\left(x_{0}, x_{1}\right)
$$

where $\left[\frac{n}{2}\right]$ stands for the greatest integer not exceeding $\frac{n}{2}$. Also, since $\beta \gamma>0$, from (3.16), we have $\left\{x_{n}\right\}_{n \in \mathbb{N}}$ is a Cauchy sequence in $X$. Since $X$ is complete, there is a point $z \in X$ such that

$$
\lim _{n \rightarrow+\infty} x_{n}=z \text {. }
$$

Let $F_{m}$ be arbitrary member of $\left\{F_{i}\right\}$. Since $\left\{x_{n}\right\} \subset F_{m}\left(x_{n-1}\right)$, by Lemma 2.6, there exists a $v_{n} \in X$ such that $\left\{v_{n}\right\} \subset F_{m}(z)$ for all $n$. Applying (3.12), we have

$$
D\left(\left\{x_{n}\right\},\left\{v_{n}\right\}\right) \leq a d\left(g\left(x_{n-1}\right), g(z)\right)+b \max \left\{d\left(g\left(x_{n-1}\right), g\left(x_{n}\right)\right), d\left(g(z), g\left(v_{n}\right)\right)\right\}
$$




$$
\begin{aligned}
& +c \max \left\{d\left(g\left(x_{n-1}\right), g(z)\right), d\left(g\left(x_{n-1}\right), g\left(x_{n}\right)\right), d\left(g(z), g\left(v_{n}\right)\right)\right\} \\
& +e \max \left\{d\left(g\left(x_{n-1}\right), g(z)\right), d\left(g\left(x_{n-1}\right), g\left(x_{n}\right)\right), d\left(g(z), g\left(v_{n}\right)\right)\right. \\
& , d\left(g\left(x_{n-1}\right), g\left(v_{n}\right)\right) \\
& +h \max \left\{d\left(g\left(x_{n-1}\right), g(z)\right), d\left(g\left(x_{n-1}\right), g\left(x_{n}\right)\right), d\left(g(z), g\left(v_{n}\right)\right)\right. \\
& \left., d\left(g\left(x_{n-1}\right), g\left(v_{n}\right)\right), d\left(g(z), g\left(x_{n}\right)\right)\right\} \\
& \leq a d\left(x_{n-1}, z\right)+b \max \left\{d\left(x_{n-1}, x_{n}\right), d\left(z, v_{n}\right)\right\} \\
& +c \max \left\{d\left(x_{n-1}, z\right), d\left(x_{n-1}, x_{n}\right), d\left(z, v_{n}\right)\right\} \\
& +e \max \left\{d\left(x_{n-1}, z\right), d\left(x_{n-1}, x_{n}\right), d\left(z, v_{n}\right), d\left(x_{n-1}, v_{n}\right)\right\} \\
& +h \max \left\{d\left(x_{n-1}, z\right), d\left(x_{n-1}, x_{n}\right), d\left(z, v_{n}\right), d\left(x_{n-1}, v_{n}\right), d\left(z, x_{n}\right)\right\}
\end{aligned}
$$

If $\lim _{n \rightarrow+\infty} v_{n} \neq z$, then letting limit as $n \rightarrow+\infty$, we have

$$
\begin{aligned}
& d\left(z, v_{n}\right) \leq(a+v+c+e+h) \max \left\{d(z, z), \max \left\{d(z, z), d\left(z, v_{n}\right)\right\}\right. \\
& \quad, \max \left\{d(z, z), d(z, z), d\left(z, v_{n}\right)\right\} \\
& \quad, \max \left\{d(z, z), d(z, z), d\left(z, v_{n}\right), d\left(z, v_{n}\right)\right\} \\
& \left.\quad, \max \left\{d(z, z), d(z, z), d\left(z, v_{n}\right), d\left(z, v_{n}\right), d(z, z)\right\}\right\} \\
& \quad<d\left(z, v_{n}\right)
\end{aligned}
$$

a contradiction. Hence

$$
\lim _{n \rightarrow+\infty} v_{n}=z
$$

Since $F_{m}$ be arbitrary, then

$$
\{z\} \subset \bigcap_{i=1}^{\infty} F_{i}(z) .
$$

\section{REFERENCES}

[1] Banach, S., Sur les operations dans les ensemles abstraits et leur application aux equations integrales, Fund. Math., 3(1922), 133-181.

[2] Beg, I., Abbas, M., Invariant approximation for fuzzy nonexpansive mappings, Mathematica Bohemica, 136(1)(2011), 51-59.

[3] Bogin, J., A generalization of a fixed point theorem og Gebel, Kirk and Shimi, Canad. Math. Bull., 19(1976), 7-12.

[4] Bose, R. K., Sahani, D., Fuzzy mappings and fixed point theorems, Fuzzy Sets \& Systems, 21(1987), 53-58.

[5] Butnariu, D., Fixed point theorems for fuzzy mappings, Fuzzy Sets \& Systems, 7(1982), 191207.

[6] Chandel, R., Ganguly, A., Bull. Calcutta Math. Soc., 32:193-198,1990.

[7] Chandra, M., Mishra, S., Singh, S., Rhoades, B.E., Coincidence and fixed points of nonexpansive type multi-valued and single-valued maps, Indian J. Pure Appl. Math.,26(5):393401,1995 .

[8] Ciric, Lj., Fixed points for generalized multi-valued contractions, Mat. Vesnik, 9:265- 272,1972.

[9] Ciric, Lj., On some nonexpansive type mappings and fixed points, Indian J. Pure Appl. Math., 24(3):145-149,1993.

[10] Gregus, M., A fixed point theorem in Banach spaces, Boll. Un. Mat. Ital.. A, 5 (1980), 193-198.

[11] Heilpern, S., Fuzzy maps and fixed point theorem, J. Math. Anal. Appl., 83(1981), 566-569.

[12] Jhade, P.K., Saluja, A. S., Kushwah, R., Coincidence \& Fixed Points of Nonexpansive Type Multi-Valued \& Single Valued Maps, European Journal of Pure And Applied Mathematics Vol. 4, No. 4, 2011, 330-339

[13] Lee, B. S., Generalized common fixed point theorems for a sequence of fuzzy mappings, Internat. J. Math. \& Math. Sci., 17(1994), 437-440. 


\section{R. D. Daheriya et al.}

[14] Mihet, D., On fuzzy contractive mappings in fuzzy metric spaces, Fuzzy Sets \& Systems, 158(2007), 915-921.

[15] Nadler Jr., S. B., Multivalued contraction mappings, Pacific J. Math., 30(1969), 475-487.

[16] Qiu, D., Schu, L., Guan, J., Common fixed point theorems for fuzzy mappings under Fcontraction condition, Chaos Solitons \& Fractals, 41(2009), 360-367.

[17] Rhoades, B. E., A common fixed point theorem for a sequence of fuzzy mappings, Interna. J. Math. \& Math. Sci., 3(1995), 447-450.

[18] Rhoades, B. E., Fixed points of some fuzzy mappings, Soochow J. Math., 22(1996), 111-115.

[19] Rhoades, B.E.,. Singh, S., Kulshrestha, C., Coincidence theorems for some multi-valued mappings, Int. J. Math. \& Math. Sci.,7:429-434,1984.

[20] Singh, K. L., Talwar, R., Fixed point of fuzzy mappings, Soochow J. Math., 19(1993), 95-102.

[21] Vijayaraju, P., Marudai, M., Fixed point theorems for fuzzy mappings, Fuzzy Sets \& Systems, 135(2003), 401-408.

[22] Watson, B., Rhoades, B. E., Fixed point for set valued mappings in metric space, Math. Japonica, 35(1990), 735-743.

[23] Watson, B., Rhoades, B.E., Generalized contraction and fixed points in metric space, Math. Japonica, 34(1989), 975-982.

[24] Weiss, M. D., Fixed points and induced fuzzy topologies for fuzzy stes, J. Math. Anal. Appl., 50(1975), 142-150.

[25] Zadeh, L. A., Fuzzy sets, Information and control, 8(1965), 338-353. 